\title{
Reconfigurable Systems in Education
}

\author{
Valery Sklyarov, Iouliia Skliarova \\ University of Aveiro, Department of Electronics and Telecommunications, IEETA, \\ 3810-193 Aveiro, Portugal \\ \{skl; iouliia\}@det.ua.pt
}

\begin{abstract}
This paper describes methods and tools that have been used for teaching disciplines dedicated to the design of reconfigurable digital systems. It demonstrates students' projects, disseminates experience in the integration of different disciplines, and gives examples of stimulating student activity. A set of animated tutorials for students that are available on WebCT with a number of practical projects that cover a variety of topics in FPGA-based design can be seen as the most valuable contribution to the area considered.
\end{abstract}

\section{Introduction}

Today, FPLDs are considered to be an alternative to ASICs, and they have already been very efficiently used in a large number of practical applications, such as coprocessors for general-purpose computers, problem-oriented digital systems, embedded controllers, and so on. Since the scope of potential applications is growing rapidly, a large number of well-prepared engineers are needed in the relevant areas. Thus, new trends must be reflected in the respective pedagogical activity of universities concerned with these subjects. Note that the domain of reconfigurable systems design is very dynamic and many-sided. Periodic upgrading of the pedagogical plans is essential in order to mirror recent advances in FPLD technology, design methods and CAD tools. Consequently, it is very important to provide an exchange of experience in pedagogical activity. This paper describes a methodology that has been used for more than 5 years for teaching reconfigurable systems at the Department of Electronics and Telecommunications of Aveiro University.

\section{Basic Directions in Teaching Reconfigurable Systems}

The major topics that have been considered as a base for the disciplines within the scope of reconfigurable systems design include design tools, prototyping boards with FPLDs and methods that provide an understanding of how FPLD-based circuits communicate with the external world, i.e. with peripheral electronic devices.

Design tools have been selected so that it is possible to learn both system-level specification languages and traditional HDL design flows. Two system-level specification languages, SystemC [1] and Handel-C [2], have been taught to students. 
SystemC is a library that permits hardware components to be modeled using a standard $\mathrm{C} / \mathrm{C}++$ compiler and it was considered just at a description level. Handel-C permits digital circuits to be described in a $\mathrm{C}$-based style and synthesized using Celoxica tools [2]. The latter together with Xilinx ISE 5.2 software [3] were used for the design, modeling, and implementation of digital systems based on FPGAs.

A number of prototyping boards have been employed for testing circuits in hardware. Recently used boards contain FPGAs from two Xilinx series; the SpartanII/Spartan-IIE (the boards TE-XC2Se [4] and RC100 [2]) and the Virtex-EM/VirtexIIPro (the PCI boards ADM-XRC and ADM-XPL [5]).

For the majority of practical applications, FPGA-based circuits have to interact with external devices. Three groups of interfaces have been studied. They are widely-used standard protocols, such as parallel, RS232, USB, etc; PCI; and those that provide interactions between FPGAs and external microchips, such as static memory, LCD controllers, microprocessors, etc.

Three kinds of applications have been proposed to students, combinatorial accelerators, hardware/software co-simulation, and processors with customized sets of instructions. During practical classes, students have to implement individual blocks for the systems mentioned above. Complete systems have to be constructed within semester projects.

The classes given to the students have a number of distinct features. The lectures are well-covered by a set of animated tutorials and examples of FPGA-targeted projects. All the required supplementary materials are available on the WebCT.

\section{Tutorials and WebCT}

In order to maximize the effectiveness of the classes, the students should have access to all the required materials. The materials can be divided into the following basic groups: manuals about FPGAs and the corresponding computer-aided design systems (ISE 5.2, ModelSim and DK1 in our case); supplementary documents (manuals on peripheral microchips, specification of interfaces, etc.); descriptions of auxiliary equipment that is required, such as logic analyzers; methods and tools that increase the productivity of education through facilities such as the extensive use of animated tutorials and providing materials for distance learning. For example, the section Tutorials in the public domain of [6] contains 10 examples. Each of them includes an animated tutorial in PowerPoint and examples of ISE 5.2 projects for VHDL-based design flow. They address the following topics:

- A sequence of steps for beginners to design, implement, and test in an FPGA, a trivial circuit based on a very simple VHDL code;

- The interaction of an FPGA with components such as LEDs, push buttons and DIP switchers through a CPLD;

- Design and functionality of a simple arithmetical circuit, which displays the results on a LCD (2 lines with 16 characters each). This tutorial explains how to use the ISE 5.2 schematic editor, the Core Generator, Xilinx libraries, hierarchical design, combining different components in the same project, interaction with an LCD controller, etc.; 
- Synthesis of finite state machines (FSM) and the use of Xilinx StateCAD;

- Synthesizable VHDL in alphabetical order. It allows any letter (for instance, $G$ Generic) to be chosen to learn the corresponding topic, to run a relevant project in ISE 5.2, to load the generated bitstream into the FPGA, and to test the circuit in hardware;

- Simulation of VHDL descriptions in ModelSim;

- Interacting with a touch panel [7] through an RS232 interface;

- Parameterizable (generic) VHDL code for a reprogrammable FSM and examples of the FSM interacting with a datapath;

- FPGAs interacting with two LCDs (4 lines 20 characters in each and 2 lines 16 characters in each). The examples explain a number of control sequences for scrolling, editing, etc.;

- A very detailed and relatively complex example that explains how to use recursive hierarchical control. It shows a $\mathrm{C}++$ program that illustrates algorithms, describes two recursive sub-algorithms, demonstrates how to construct a recursive hierarchical FSM that builds a special binary tree from a sequence of arbitrary integers and sorts integers on the basis of this binary tree. The results (i.e. the sorted data) are displayed on an LCD.

All the tutorials make use of different animation effects available in PowerPoint (Windows XP). This enables many processes to be demonstrated in a step by step manner, such as all the events appearing in each clock cycle; how VHDL code activates these events and reacts; how various bits in interface lines are changed, etc.

Similar tutorials have been prepared for explaining the functionality of FPGA DLLs, demonstrating an interaction with a mouse, a keyboard, a VGA monitor, and for a number of Handel-C topics. They are available in English and in Portuguese.

\section{Stimulation of Student Activity and Integration with other Disciplines}

Two types of evaluation have been proposed to the students. The first is a traditional examination. The examination can be replaced by the second type of evaluation through an individual project that is suggested in the middle of a semester. According to the requirements, students have to design, implement, and test a digital system based on commercially available FPGAs. Potential projects are discussed with the students. This allows a task to be chosen from the area that is of the most interest to a particular student. The results of the projects have to be demonstrated in a working FPGA-based device and presented in a written report before the end of the examination period. The best projects are recommended for publications in the magazine "Electrónica e Telecomunicações" that is issued by the Department. All these publications can be accessed through the WebCT [6].

The methodology provides a very important opportunity, especially for final year students. It permits integration between different disciplines to be established. The group of disciplines considered in this paper suggests methods and tools; the other groups of disciplines offer applications. This approach provides additional motivation 
to the students because reconfigurable systems can be linked with practical work in other disciplines that particular students are interested in.

Our experience has shown that there are some auxiliary methods that stimulate the work of students. First of all, the result of the work should be visible and touchable especially at the beginning. That is why it is reasonable to use stand-alone boards that are cheap and provide a number of interactions such as communication between an FPGA and a mouse, a keyboard, a VGA monitor, LCD panels, etc. Only after some period of time hidden PCI-based prototyping boards can be used. As a rule they are much more expensive and do not permit the results to be appreciated visually. The work is organized through a set of API functions and it looks like programming. On the other hand, PCI-based boards contain much more powerful FPGAs and they are recommended for experienced students, especially those in Ph.D. and M.Sc. scholarships.

For example, in 2002/2003 a combinatorial processor that implements an exact algorithm for solving the covering problem has been proposed as a project for final year students. Initially, a stand-alone RC100 prototyping board with an FPGA from the Spartan-II family was used. All the individual components of the project were described in Handel-C and carefully tested using available peripheral devices and drivers supplied by Celoxica. Finally the entire circuit was implemented. After that the same circuit was constructed using the ADM-XPL PCI board [5] containing FPGA XC2VP7 of Virtex-II Pro family. This circuit allows much more complicated problems to be resolved [8]. The previous experience gained with a relatively cheap stand-alone board provides a basis for achieving results rapidly, and very similar methods and tools can be used for the most advanced FPGAs available on the market.

\section{Conclusion}

This paper disseminates experience in teaching reconfigurable systems, summarizes the pedagogical methods that have been adopted, the organization of classes, the basic directions of student's projects, and many other aspects. This work was supported by the grants FCT-PRAXIS XXI/BD/21353/99 and POSI/43140/CHS/2001.

\section{References}

SystemC. [Online]. Available: http://www.systemc.org/

2. Handel-C, DK1, RC100. [Online]. Available: http://www.celoxica.com/

3. ISE 5.2, Xilinx series FPGA. [Online]. Available: http://www.xilinx.com/

4. Spartan-IIE Development Platform. [Online]. Available: www.trenz-electronic.de

5. Alpha Data. [Online]. Available: http://www.alpha-data.com

6. http://webct.ua.pt, "2 semester", the discipline "Computação Reconfigurável”, public domain is indicated by the letter " $i$ " enclosed in a circle. Login and password for access to the protected section can also be provided (via e-mails: skl@,ieeta.pt, iouliia@det.ua.pt )

7. EA KIT240-7, EA DIP204-4. Electronic Assembly. Available: http://www.lcd-module.de

8. Sklyarov, V., Skliarova, I. Almeida, P., Almeida, M.: High-Level Design Tools for FPGA-based Combinatorial Accelerators. Proceedings of FPL'2003 (Lisbon, 2003) 